\title{
Terminal Flow of Cluster-Forming Supramolecular Polymer Networks: Single-Chain Relaxation or Micelle Reorganization?
}

\author{
Anton Mordvinkin, ${ }^{1}$ Diana Döhler, ${ }^{2}$ Wolfgang H. Binder, ${ }^{2}$ Ralph H. Colby, ${ }^{3}$ and Kay Saalwächter ${ }^{1, *}$ \\ ${ }^{1}$ Institut für Physik-NMR, Martin-Luther-Universität Halle-Wittenberg, Betty-Heimann-Straße 7, 06120 Halle (Saale), Germany \\ ${ }^{2}$ Institut für Chemie-Makromolekulare Chemie, Martin-Luther-Universität Halle-Wittenberg, \\ von-Danckelmann-Platz 4, 06120 Halle (Saale), Germany \\ ${ }^{3}$ Materials Science and Engineering, The Pennsylvania State University, University Park, Pennsylvania 16802, USA
}

(Received 5 June 2020; accepted 18 August 2020; published 17 September 2020)

\begin{abstract}
We correlate the terminal relaxation of supramolecular polymer networks, based on unentangled telechelic poly(isobutylene) linear chains forming micellar end-group clusters, with the microscopic chain dynamics as probed by proton NMR. For a series of samples with increasing molecular weight, we find a quantitative agreement between the terminal relaxation times and their activation energies provided by rheology and NMR. This finding corroborates the validity of the transient-network model and the special case of the sticky Rouse model, and dismisses more dedicated approaches treating the terminal relaxation in terms of micellar rearrangements. Also, we confirm previous results showing reduction of the activation energy of supramolecular dissociation with increasing molecular weight and explain this trend with an increasing elastic penalty, as corroborated by small angle x-ray scattering data.
\end{abstract}

DOI: 10.1103/PhysRevLett.125.127801

Supramolecular polymer networks with reversible crosslinking sites constitute a relatively new class of smart materials that are susceptible to various external stimuli to perform desired tasks such as self-healing and shape memory [1-4]. The final macroscopic properties of the materials (e.g., mechanical stability and self-healing ability) are known to be defined by their microstructure. Up to now, a multitude of models have been designed to describe mechanical behavior of supramolecular polymer networks based on rheological data [5-11] and x-ray scattering $[12,13]$. While most models are chain based and assume cross-linking sites of defined and low functionality, the incompatibility of sticky and polar end groups with the often unpolar polymer backbones leads to the formation of much larger end-group aggregates in many if not most cases. We here focus on unentangled telechelic poly (isobutylene)-based (PIB-based) networks with cross-linking junctions in the form of flowerlike micelles incorporating a large number of the barbituric acid end groups (stickers). This system should comply with the only available dedicated micellar model developed by Semenov and co-workers [6,7], which we here contrast with the transient-network (TN) model [14,15] and a special case of the sticky Rouse (SR) model [8].

The TN/SR models both predict a plateau in the storage modulus caused by the network formation, which persists on the timescale shorter than the effective bond lifetime of the stickers $\left(\tau_{\mathrm{st}}^{*}\right)$. Once $\tau_{\mathrm{st}}^{*}$ is reached, stickers break free and start performing hopping motions from micelle to micelle, mediated by single chains, leading to terminal flow [see Fig. 1(a)]. Stress relaxation is thus governed by single chains. The activation energy of the process is contributed by coupled dissociation of a single sticker and segmental motion. In contrast, the micellar model suggests that a jammed packing of repulsive micelles (sketched as gray circles in Fig. 1) prevents flow, and that terminal flow is only possible upon dissolution and reorganization of whole micelles realized via simultaneous debridging of all chains emanating from the micelle, followed by hopping of the

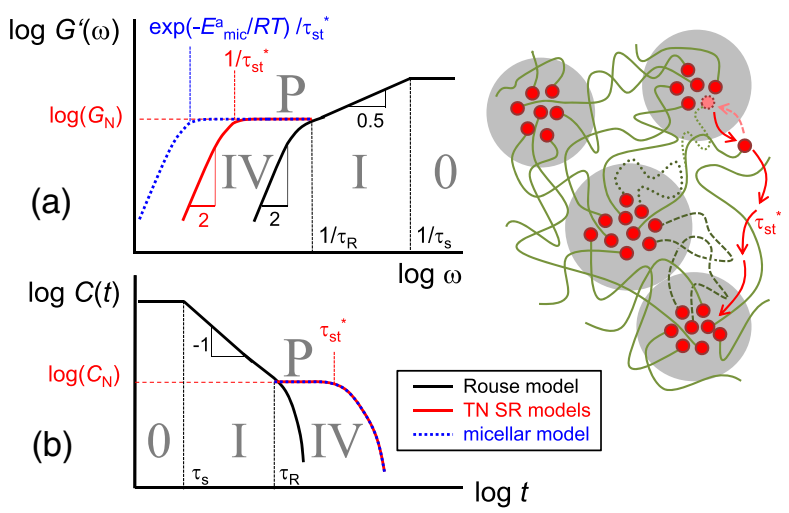

FIG. 1. (a) Storage modulus $G^{\prime}$ and (b) the orientation autocorrelation function $C(t)$ following the predictions of the Rouse (black), TN SR (red), and micellar (blue) models. The specified dynamic regimes are 0, glassy; I, Rouse; P, plateau; IV, terminal flow. The sketch of the network structure includes a single-chain relaxation process (debridging on a timescale $\tau_{\mathrm{st}}^{*}$ ), two topologically connected loops (dashed), and an elastically inactive loop (dotted). The repulsive micellar coronas are indicated as gray background circles. 
micelle to a new position [6,7]. This leads to a relaxation time which is exponentially larger than the relaxation time of a single chain, $\tau_{\mathrm{st}}^{*} \exp \left(E_{a}^{\mathrm{mic}} / R T\right)$, where $E_{a}^{\mathrm{mic}}$ is the relevant activation energy including contributions of the deformation energy of a micelle and its neighborhood and micellar diffusion. The frequency dependence of the shear storage modulus predicted by the two models can be seen in Fig. 1(a). The Rouse model prediction for the case of nonassociating unentangled polymer chains is depicted for comparison.

A recent study of micelle-forming telechelic PIBs similar to the ones investigated herein showed that the high viscosity of a system of singly functionalized chains is indeed best described by models assuming a colloidal suspension of micelles [12]. However, the factors that govern the onset of terminal flow in a bifunctional system featuring an elastic plateau are yet to be elucidated. To decide which of the models applies, an experimental method, complementary to rheology and sensitive to single-chain dynamics, is required. In this regard, solid-state NMR can be readily utilized [16,17], whereas neutron scattering techniques [18] do not reach the required timescale in the ms range.

Here, we used a recently developed data analysis approach of multiple-quantum (MQ) NMR data to extract of bond lifetimes on a molecular scale [16]. The fitting of the data delivers a part of the segmental orientation autocorrelation function $(\mathrm{OACF})$ of the second Legendre polynomial $C(t)=5\left\langle P_{2}[\cos \theta(t)] P_{2}[\cos \theta(0)]\right\rangle$ [19], where $\theta$ is the segmental orientation with respect to the external magnetic field. $C(t)$ is used to quantify the polymer chain dynamics. It is approximated to decay within the analyzed time interval according to a power law with exponent $\kappa$ $[16,20]$ starting from a certain anisotropy level [amplitude of $\left.C(t) \propto D_{\text {res }}^{2}\right]$ defined by dipolar couplings $D_{\text {res }}\left(\tau_{\mathrm{DQmin}}\right)$, where $\tau_{\mathrm{DQmin}}$ is the first DQ data point marking the beginning of the analyzed time interval. The exponent $\kappa$, i.e., the slope of $\log C(t)$ vs $\log t$, is henceforth just referred to as "slope of $C(t)$." The approach has been validated and applied to a more complicated case of entangled ionic networks [17], where the effective bond lifetime could be correlated with an extra relaxation seen in dynamic mechanical analysis. As a result, the single-chain mechanism assumed in the sticky reptation model could be validated, but the terminal relaxation remained elusive due to the large chain length.

$C(t)$ for the case of unentangled associating polymer chains and their nonassociating counterparts is sketched in Fig. 1(b). The case of unassociating polymers can be well described by the Rouse model, defining the shortest relaxation time to be a segmental relaxation time $\tau_{s}$, below which no relevant dynamics is assumed to take place (regime 0 according to the Doi-Edwards classification [21]). Starting from $\tau_{s}$ up to the Rouse relaxation time $\tau_{R}$, subdiffusive free segmental motions take place (Rouse regime, or regime I), after which a polymer chain moves diffusively as a whole object (regime IV). In regime I, $C(t)$ decays with an exponent of -1 followed in regime IV by an exponential decay. Note that the regime-I exponent is typically found in the range between -0.7 and -0.9 due to too few Rouse modes available [22,23], in good agreement with simulations [24].

The TN/SR and micellar models for associating polymer chains in turn predict the appearance of a rubbery plateau due to the formation of a supramolecular network followed by terminal flow at $\tau_{\mathrm{st}}^{*}$, as was mentioned above. However, in contrast to the mentioned differences in stress relaxation, $C(t)$ of the two models must show similar decays, as $C(t)$ is intrinsically sensitive to single-chain relaxation. Thus, matching or nonmatching terminal relaxation times (and activation energies) measured by MQ NMR and rheology will provide a distinction between the TN/SR and micellar models, respectively.

As model systems, we used PIBs of increasing molecular weight $\left(M_{n}=4,7.9,13.8 \mathrm{kDa}\right.$, with $\mathrm{Ð}=1.3,1.2,1.2$, respectively) modified on both ends with barbituric acid (BA), designated as $4 k \mathrm{BA} 2,8 k \mathrm{BA} 2$, and $14 k \mathrm{BA} 2$, respectively. For the chemical structure and further experimental details we refer to the Supplemental Material (SM) [25]. The samples were already investigated previously in a number of works [13,26,27] and were found to build networks of interconnected micelles, as validated by small-angle x-ray scattering (SAXS) and rheological measurements. To check reproducibility, we repeated the rheological and SAXS measurements (see Figs. 2 and SM1 [25], respectively) on in part newly synthesized samples, and confirmed the findings in Refs. [13,27].

Results of the SAXS analyses are provided in Table I (see SM [25] for details). The aggregation numbers $A N$ estimated by using space-filling (SF) arguments as well as a Percus-Yevick (PY) hard-sphere model for repulsive micelles are in good mutual agreement. The interaggregate distance $r_{c c}$ increases with the molecular weight less strongly as compared to the expected increase of the end-to-end distance $R_{E E}$ of an ideal Gaussian chain. This trend lets us conclude that network chains tend to be increasingly compressed, which could lead to an additional elastic penalty causing an effective weakening of supramolecular interactions.

To extract rheological relaxation times, we constructed apparent master curves focusing on the terminal regime, as discussed previously [17], where the dynamics are governed by additional thermal activation of the sticky end groups rather than the PIB backbone alone. The procedure relies on the temperature dependence of the relevant segmental or chain relaxation times, where a shift factor $a_{T}$, being a ratio of relaxation times at two different temperatures, can be used to superpose the rheological data taken at these two temperatures along the logarithmic frequency $(\log \omega)$ axis [28]. We then performed simultaneous fits on $G^{\prime}$ and $G^{\prime \prime}$ data based on the Maxwell model 

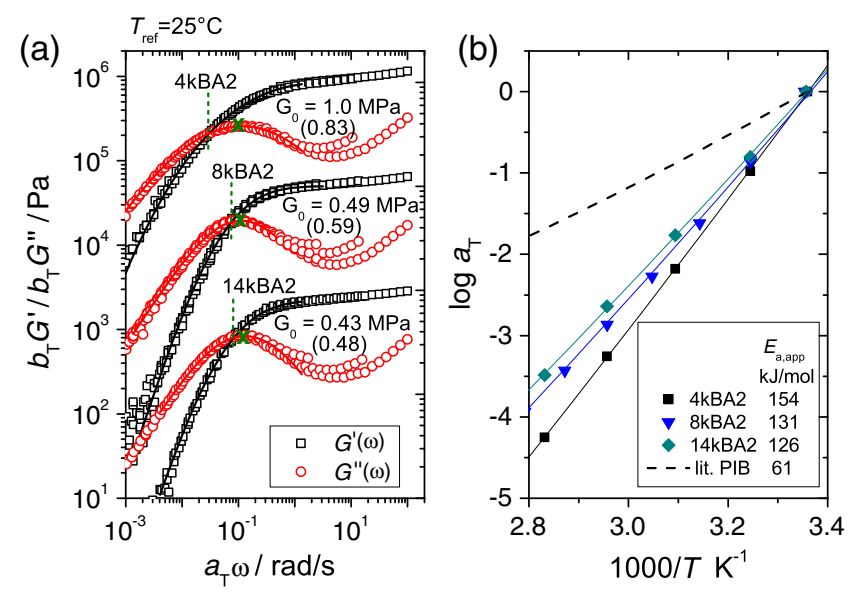

FIG. 2. (a) Apparent rheological master curves $\left(G^{\prime}\right.$ and $\left.G^{\prime \prime}\right)$ for the telechelic samples at $T_{\text {ref }}=25^{\circ} \mathrm{C}$. The solid lines are the fits based upon eqs. (1) and (2), respectively, with results for the modulus $G_{0}$ indicated (the values in brackets are the affine predictions). The dashed lines indicate the modulus crossovers, while the crosses indicate the fitted inverse median $\tau_{\mathrm{st}}^{*}$. The data of $8 k \mathrm{BA} 2$ and $14 k \mathrm{BA} 2$ are vertically shifted downwards by 1 and 2 decades, respectively, for clarity. (b) Rheological shift factors $a_{\mathrm{T}}$ for the telechelic samples, covering only the sticker-dominated regime. The segmental $a_{\mathrm{T}}$ for pure PIB taken from literature [28] is also given for reference. The curves represent fits based on the product of the Vogel-Fulcher and Arrhenius temperature dependencies.

with a log-normal distribution of relaxation times [see Fig. 2(a)]. The equations for the simultaneous fit read

$$
G^{\prime}(\omega)=\int P\left[\ln \left(\tau_{\mathrm{st}}^{*}\right)\right] G_{0} \frac{\left(\tau_{\mathrm{st}}^{*} \omega\right)^{2}}{1+\left(\tau_{\mathrm{st}}^{*} \omega\right)^{2}} d \ln \left(\tau_{\mathrm{st}}^{*}\right)
$$

and

$$
G^{\prime \prime}(\omega)=\int P\left[\ln \left(\tau_{\mathrm{st}}^{*}\right)\right] G_{0} \frac{\tau_{\mathrm{st}}^{*} \omega}{1+\left(\tau_{\mathrm{st}}^{*} \omega\right)^{2}} d \ln \left(\tau_{\mathrm{st}}^{*}\right)
$$

where $P\left[\ln \left(\tau_{\mathrm{st}}^{*}\right)\right]$ is the $\log$-normal distribution density function of $\tau_{\mathrm{st}}^{*}, G_{0}$ is the plateau modulus. For the studied samples, the logarithmic distribution width of $\tau_{\mathrm{st}}^{*}$ was found to be $0.9-1.7$ decades, which is substantial and excludes the use of a simple Maxwell model (see SM [25] for details). In fact, the fitted median $\tau_{\mathrm{st}}^{*}$ deviates from the inverse frequencies at the modulus crossover by up to a factor of 4.

TABLE I. Results of SAXS experiments.

\begin{tabular}{lcccc}
\hline \hline Sample & $A N_{\mathrm{SF}}$ & $A N_{\mathrm{PY}}$ & $r_{c c} / \AA$ & $R_{E E} / \AA$ \\
\hline $4 k \mathrm{BA} 2$ & 59 & 49 & 58.2 & 54.4 \\
$8 k \mathrm{BA} 2$ & 49 & 35 & 66.8 & 73.5 \\
$14 k \mathrm{BA} 2$ & 44 & 38 & 77.6 & 97.1 \\
\hline \hline
\end{tabular}

Results for $G_{0}$, which decrease according to the increasing chain length, are listed along with the curves in Fig. 2(a), including in brackets theory estimates based upon the affine model of rubber elasticity including the entanglement-related plateau modulus $\left(G_{0, \mathrm{th}}=G_{e}+\left[\rho R T / M_{w}\right]\right)$. Considering the significant experimental uncertainties related to using small $(3 \mathrm{~mm})$ plates, the values are in good mutual agreement. This is remarkable, because we expect that not all chains form bridges but also loops (see the sketch in Fig. 1). Based upon our previous application [17] of the scaling arguments of Semenov and Rubinstein [7] for unperturbed chains, the bridging chain fractions are estimated to 0.58 , 0.67 , and 0.70 for samples $4 k \mathrm{BA} 2,8 k \mathrm{BA} 2$, and $14 k \mathrm{BA} 2$, respectively. We thus cannot exclude a cancellation of effects, related to the micellar aggregates acting also as "soft filler," providing volumetric reinforcement. On the other hand, we were not able to detect a significant separable fraction of isotropically moving chains by NMR, as expected for loops. We thus suspect that even in the $4 k$ system, most loops may be topologically connected to other loops, consequently increasing the number of effective bridges.

Turning to NMR results, MQ NMR data were obtained using a combination of two different pulse sequences to cover the full temperature range [23,29], see SM [25] for details. The MQ NMR signal functions-a double-quantum (DQ) signal from coupled protons and a fully dipolar refocused MQ decay signal-were fitted simultaneously to an analytical formula that assumes a power-law $C(t)$ in the interval $\tau_{\mathrm{DQmin}} \cdots \tau_{\mathrm{DQmax}}$ [16]. Such fits providing as relevant parameters the amplitude ( $\propto D_{\text {res }}^{2}$ ) and exponent $\kappa$ of an isothermal piece of $C(t)$ are exemplarily shown in Fig. 3 for $4 k \mathrm{BA} 2$ at $70^{\circ} \mathrm{C}$. It can be seen that the fitting curves do not show a perfect agreement with the data. This originates from a distribution of $D_{\text {res }}$ caused by the disordered structure and the broad distribution of $\tau_{\mathrm{st}}^{*}$.

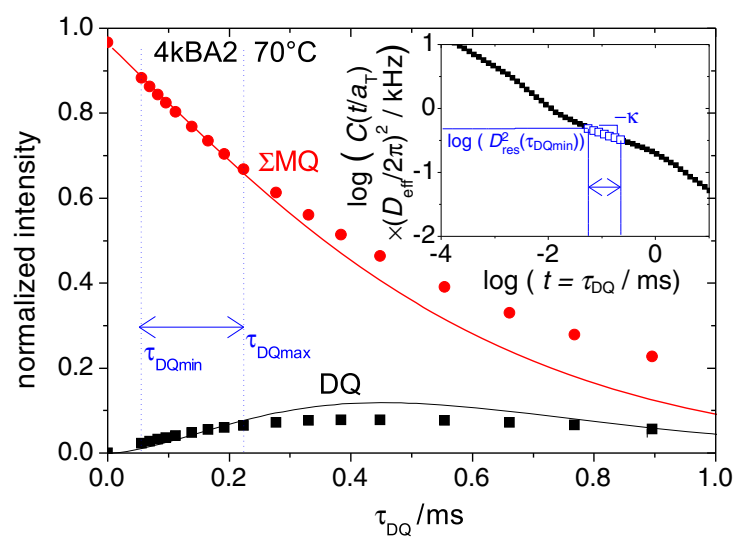

FIG. 3. MQ NMR data obtained for the $4 k \mathrm{BA} 2$ sample at $70^{\circ} \mathrm{C}$. The displayed curves correspond to the simultaneous fits according to the assumption of a power-law decay of $C(t)$ in the fitted time interval (marked by dotted blue lines). The extracted $C(t)$ part is shown in the inset along with data from other temperatures (black solid symbols). 

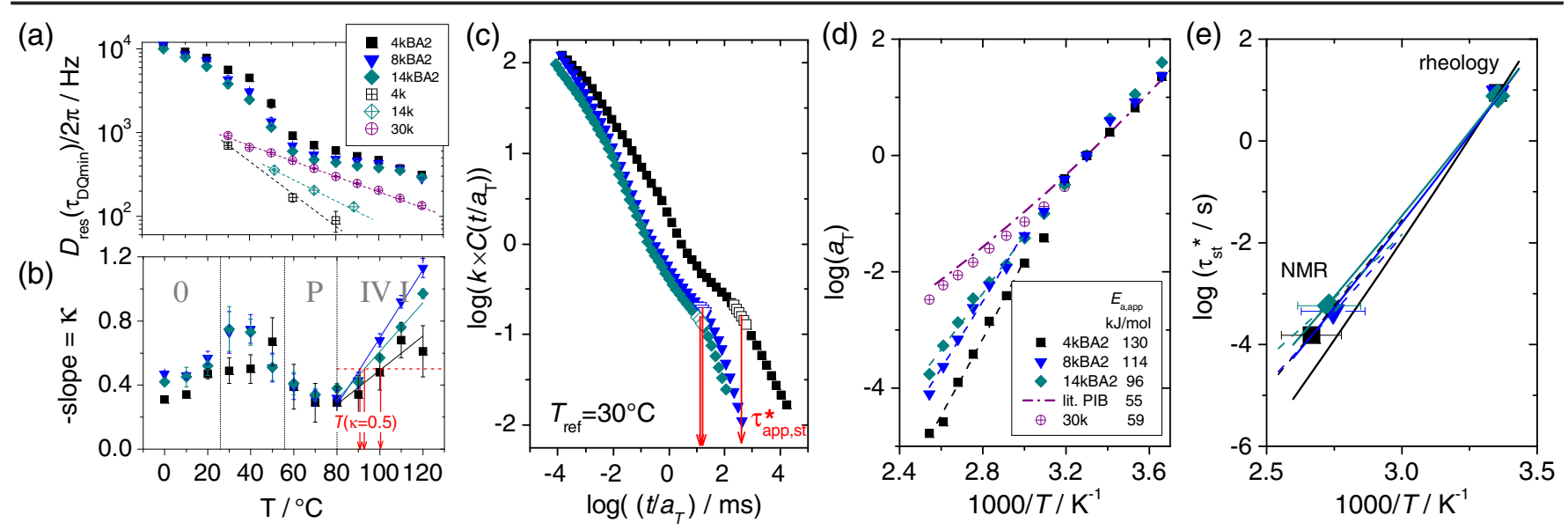

FIG. 4. (a) $C(t)$ amplitudes and (b) slopes in the form of MQ NMR parameters $D_{\text {res }}\left(\tau_{\mathrm{DQmin}}\right)$ and $\kappa$, respectively. Temperatures at which $\kappa=0.5$ are marked. (c) Amplitude-normalized apparent $C(t)$ master curves reduced to $T_{\text {ref }}=30^{\circ} \mathrm{C}$. Apparent values of sticky bond lifetimes $\tau_{\text {st,app }}^{*}$, determined as centers of time intervals at temperatures at which $\kappa=0.5$, are specified. (d) Shift factors obtained from the construction of the apparent $C(t)$ master curves for the supramolecular samples. The shift factors of the PIB polymer melt (30k) are given for comparison. (e) Temperature dependencies of the terminal flow relaxation times obtained by NMR and rheology. In both Arrhenius plots, the solid and dashed lines correspond to fits of the shift factors from rheology and NMR, respectively.

In fact, a minor fraction (up to 10\%) of significantly more constrained segments around the micellar aggregates can be estimated from bimodal fits of the NMR data in the plateau region (it is, however, notable that we do not detect any significant fraction of isotropically mobile, non-loadbearing strands as sketched in Fig. 1). Any such distributions were neglected in the fits, forcing the response to be captured with a single power law $C(t)$. Nevertheless, the extracted values of $D_{\text {res }}\left(\tau_{\mathrm{DQmin}}\right)$ and $\kappa$ represent reliable averages.

At this point, we need to explain how to obtain $\tau_{\mathrm{st}}^{*}$ and justify why we apply a fitting model based upon a power-law $C(t)$, whereas a Maxwell model with log-normal distribution, implying multiexponential relaxation, was suitable to describe the mechanical results. As discussed in SM [25], reliable and stable fits to NMR data on the basis of an exponential $C(t)$ were precluded by the significant dynamic heterogeneity. We thus chose to stick to the power-law model (which implies a distribution of exponential relaxations), and determined $\tau_{\mathrm{st}}^{*}$ values as arithmetic averages over the fitting interval, $\tau_{\mathrm{st}}^{*}=\left(\tau_{\mathrm{DQmin}}+\tau_{\mathrm{DQmax}}\right) / 2$ at the temperatures at which $C(t)$ took on a fitted power-law slope of -0.5 in the region of the terminal flow. The fitted apparent power-law slope of the multiexponential $C(t)$ depends on the distribution width of $\tau_{\mathrm{st}}^{*}$, which is significant according to the rheology results discussed above.

The $C(t)$ amplitudes and slopes extracted from the fits, as well as the apparent $C(t)$ master curves constructed from the piecewise power laws for all the samples can be seen in Figs. 4(a) and 4(b). $D_{\text {res }}\left(\tau_{\mathrm{DQmin}}\right)$ of the azide-telechelic PIB precursors decreases with temperature, as predicted by the Rouse model. In the case of the barbiturate-modified samples, in turn, we observe (i) larger $D_{\text {res }}\left(\tau_{\mathrm{DQmin}}\right)$ values which first decay to a plateau before they further decrease and (ii) $\kappa$ values that first decrease beyond regime I and then increase again. This observation suggests that $C(t)$ has a plateau followed by a terminal flow, similar to the rheological storage modulus. In the region of the terminal flow, we can find by a linear interpolation the temperature at which $\kappa=0.5$ and, as noted above, take the arithmetic average of the fitting time interval at this temperature (or closest studied temperature) to be our $\tau_{\mathrm{st}}^{*}$.

In the next step, we aimed to obtain the temperature dependencies of the found $\tau_{\mathrm{st}}^{*}$. This was accomplished via the master curve construction assuming the validity of the time-temperature superposition principle. In Figs. 4(c) and 4(d), one can see the resulting master curves and the corresponding horizontal shift factors $a_{T}$, respectively, to be compared with the rheology results in Fig. 2. It can be seen that the $C(t)$ master curves do not overlap in the Rouse regime owing to small variations in the glass transition temperature. It is important to stress that the $\tau_{\mathrm{st} \text {,app }}^{*}$ marked in Fig. 4(c) are apparent values in that they cannot be compared among the samples. This is due to limitations inherent to constructing apparent master curves in a thermorheologically complex system, as discussed previously [17]. For this reason, we focus below on the isothermal values as described above. The shift factors first follow the temperature dependence of the segmental shift factor of PIB taken from literature [28], and start to deviate above $50^{\circ} \mathrm{C}$, where $\tau_{\mathrm{st}}^{*}$ starts to control the detected larger-scale motions [17].

The shift factors in the high-temperature regions were fitted with the product of the Vogel-Fulcher temperature dependence, corresponding to the segmental dynamics of pure PIB, and an assumed Arrhenius temperature dependence of the supramolecular dissociation [5,17]. The obtained apparent activation energies $\left(E_{a, \text { app }}\right)$, being the 
sum of the linearized segmental values in the studied temperature range and the supramolecular dissociation energies, are given in the legend of Fig. 4d. The $E_{a \text { app }}$ values were found to be around $20 \%$ lower than the corresponding quantities provided by rheology. This is not surprising in the light of large errors of the NMR values (about 30\%) due to the crude power-law approximation of $C(t)$. The values decrease with increasing molecular weight, which we attribute to the mentioned compression of network chains causing an elastic penalty and, as a result, an effective reduction of the effective interaction energy. Since classical as well as potential nonclassical contributions to chain elasticity are athermal [30] and would not affect $E_{a \text {,app }}$, more subtle effects related to chain packing and free volume near the clustered stickers may be relevant.

In Fig. 4(e), we plotted the terminal flow relaxation times isothermally determined by NMR and rheology along with the temperature dependencies taken from the two sets of shift factors. It can be seen that in all cases NMR and rheological results agree nearly quantitatively, which lets us finally conclude that the dynamics of supramolecular networks built by telechelic chains forming micelles with large aggregation numbers can be well described by the TN/SR models. Hence, the dedicated micellar model does not appear to apply in the studied case.

In summary, we have applied a combination of rheology and MQ NMR to clarify the molecular origin of the terminal relaxation of supramolecular networks comprising interconnected micellar aggregates. To this end, we compared the relaxation times and their activation energies obtained from the two methods. As a result, we were for the first time able to show that the timescale for macroscopic terminal relaxation of a supramolecular network is the same as the average timescale of relaxation of each and every single chain. Thus, we could validate that the transientnetwork or sticky Rouse models can be utilized for describing polymer chain dynamics and stress relaxation. A micellar model based upon collective dynamics, requiring simultaneous debridging of all chains, would predict a much longer flow time in rheology and is thus not applicable. An energetically more favorable alternative to the detachment and reattachment of single chains may be a mechanism of cluster merging and dissociation, as recently observed in computer simulations [31]. More research will be required to decide which detailed mechanism is most feasible in the given case of comparably large clusters.

K. S. thanks all members of the Colby research group and Nichole Wonderling of the MCL for their generous help concerning the rheological and SAXS experiments during his sabbatical. We also thank Florian Herbst for his contributions to the syntheses. Funding was provided by the DFG through Grants No. SA982/9-1 and No. BI 1337/ 7-2 within the Priority Programme SPP 1568 "Design and
Generic Principles of Self-Healing Materials," and the "Leistungszentrum Chemie und Biosystemtechnologie" of the state of Saxony-Anhalt.

kay.saalwaechter@physik.uni-halle.de; http:www.physik .uni-halle.de/nmr

[1] Self-Healing Polymers: From Principles to Applications, edited by W. H. Binder (Wiley-VCH, Weinheim, 2013).

[2] Supramolecular Polymer Networks and Gels, edited by S. Seiffert (Springer, Switzerland, 2015).

[3] Self-Healing Materials, edited by M. D. Hager, S. v. d. Zwaag, and U.S. Schubert (Springer, Switzerland, 2016).

[4] A. Campanella, D. Döhler, and W. H. Binder, Macromol. Rapid Commun. 39, 1700739 (2018).

[5] L. Leibler, M. Rubinstein, and R. Colby, Macromolecules 24, 4701 (1991).

[6] A. N. Semenov, J.-F. Joanny, and A. Khokhlov, Macromolecules 28, 1066 (1995).

[7] N. Semenov and M. Rubinstein, Macromolecules 35, 4821 (2002).

[8] Q. Chen, G. J. Tudryn, and R. H. Colby, J. Rheol. 57, 1441 (2013).

[9] M. Ahmadi, L. G. D. Hawke, H. Goldansaz, and E. v. Ruymbeke, Macromolecules 48, 7300 (2015).

[10] Q. Chen, Z. Zhang, and R. H. Colby, J. Rheol. 60, 1031 (2016).

[11] M. J. Mateyisi, J.-U. Sommer, K. K. Müller-Nedebock, and G. Heinrich, J. Chem. Phys. 148, 244901 (2018).

[12] T. Yan, K. Schröter, F. Herbst, W. H. Binder, and T. Thurn-Albrecht, Macromolecules 47, 2122 (2014).

[13] T. Yan, K. Schröter, F. Herbst, W. H. Binder, and T. Thurn-Albrecht, Macromolecules 50, 2973 (2017).

[14] M. S. Green and A. V. Tobolsky, J. Chem. Phys. 14, 80 (1946).

[15] F. Tanaka and S. F. Edwards, Macromolecules 25, 1516 (1992).

[16] A. Mordvinkin and K. Saalwächter, J. Chem. Phys. 146, 094902 (2017); 148, 089901(E) (2018).

[17] A. Mordvinkin, M. Suckow, F. Böhme, R. H. Colby, C. Creton, and K. Saalwächter, Macromolecules 52, 4169 (2019).

[18] M. Monkenbusch, M. Krutyeva, W. Pyckhout-Hintzen, W. Antonius, C. H. Hövelmann, J. Allgaier, A. Brás, B. Farago, A. Wischnewski, and D. Richter, Phys. Rev. Lett. 117, 147802 (2016).

[19] F. Vaca Chávez and K. Saalwächter, Macromolecules 44, 1549 (2011).

[20] K. Saalwächter and A. Heuer, Macromolecules 39, 3291 (2006).

[21] M. Doi and S. F. Edwards, The Theory of Polymer Dynamics (publisher Oxford University Press, New York, 1986).

[22] A. Herrmann, V. N. Novikov, and E. A. Rössler, Macromolecules 42, 2063 (2009).

[23] M.-L. Trutschel, A. Mordvinkin, F. Furtado, L. Willner, and K. Saalwächter, Macromolecules 51, 4108 (2018).

[24] Z. Wang, A. E. Likhtman, and R. G. Larson, Macromolecules 45, 3557 (2012). 
[25] See Supplemental Material at http://link.aps.org/supplemental/ 10.1103/PhysRevLett.125.127801 for experimental details, SAXS analyses and discussion of the fitting models.

[26] F. Herbst, S. Seiffert, and W. H. Binder, Polym. Chem. 3, 3084 (2012).

[27] T. Yan, K. Schröter, F. Herbst, W. H. Binder, and T. ThurnAlbrecht, Sci. Rep. 6, 32356 (2016).
[28] J. D. Ferry, Viscoelastic Properties of Polymers (John Wiley and Sons, Toronto, 1980).

[29] K. Saalwächter, Prog. NMR Spectrosc. 51, 1 (2007).

[30] F. T. Oyerokun and K. S. Schweizer, J. Chem. Phys. 120, 9359 (2004).

[31] D. Amin, A. E. Likhtman, and Z. Wang, Macromolecules 49, 7510 (2016). 